\title{
Development of a computed cleansing score to assess quality of bowel preparation in colon capsule endoscopy
}

\section{(ㄷ)(구웅}

Authors

Aymeric Becq $^{1,2}$, Aymeric Histace ${ }^{3}$, Marine Camus ${ }^{1,2}$, Isabelle Nion-Larmurier ${ }^{1}$, Einas Abou Ali ${ }^{1}$, Olivia Pietri ${ }^{1,2}$, Olivier Romain $^{3}$, Ulriikka Chaput ${ }^{1}$, Cynthia Li ${ }^{1,4}$, Philippe Marteau ${ }^{1,2}$, Christian Florent ${ }^{1,2}$, Xavier Dray ${ }^{1,2,3}$

Institutions

1 Saint-Antoine Hospital, AP-HP, Department of Hepatogastroenterology, 184 rue du Faubourg Saint Antoine, 75012 Paris, France

2 Sorbonne University, Paris, France

3 ETIS UMR 8051, University Paris-Seine, University of Cergy-Pontoise, ENSEA, CNRS, Cergy, France

4 College of Arts and Sciences, Drexel University, Philadelphia, Pennsylvania, United States

submitted 10.8.2017

accepted after revision $\quad 27.12 .2017$

Bibliography

DOI https://doi.org/10.1055/a-0577-2897 |

Endoscopy International Open 2018; 06: E844-E850

(C) Georg Thieme Verlag KG Stuttgart · New York

ISSN 2364-3722

Corresponding author

Xavier Dray, MD, PhD, Department of Digestive Diseases, Saint-Antoine Hospital - APHP, 184 rue du Faubourg SaintAntoine, 75571 Paris cedex 12, France

Fax: +0033-1-49-28-29-70

xavier.dray@aphp.fr

\section{ABSTRACT}

Background and study aims Colon capsule endoscopy (CCE) does not possess an objective and reliable scoring system to assess the quality of visualization of the colon mucosa. The aim of this study was to establish a colonic computed assessment of cleansing (CAC) score able to discriminate "adequately cleansed" from "inadequately cleansed" CCE still frames.

Patients and methods Twelve normal and complete CCEs, using the Pillcam Colon 2 system (Medtronic, Minnesota, United States), were prospectively selected amongst a database. A CAC score, defined as the ratio of color intensities red over green (R/G ratio), and red over brown (R/(R+ $\mathrm{G})$ ratio) was calculated for each extracted colonic frame. After sorting and random selection, two sets of still frames representative of the range of these ratios were obtained. These images were analyzed twice in random order by two experienced CCE readers who were blinded to the CAC scores. A receiver operating characteristic (ROC) curve was forged for both types of ratios and a threshold established, yielding the highest diagnostic performance in terms of adequate cleansing assessment.

Results Four-hundred-and-eight frames were extracted. Regarding the R/G ratio, a threshold value of 1.55 was calculated, with a sensitivity of $86.5 \%$ and a specificity of $77.7 \%$. Regarding the $R /(R+G)$ ratio, a threshold value of 0.58 was calculated with a sensitivity of $95.5 \%$ and a specificity of $62.9 \%$.

Conclusion The two proposed CAC scores based on the ratio of color intensities come with high sensitivities for discriminating between "adequately cleansed" and "inadequately cleansed" CCE still frames, but they lack specificity. Further refinement, with implementation of additional image parameters, is warranted.

\section{Introduction}

Colon capsule endoscopy (CCE) was first used in 2006 (PillCam Colon, Medtronic, Minnesota, USA) [1 - 3] and a second generation (PillCam Colon 2, Medtronic) was developed and launched in 2009 [4]. The main goal of CCE is to detect colorectal polyps and is of particular interest in patients who decline, are at elevated risk of complications, or have had an incomplete colonoscopy [5-8]. Advantages of this procedure are that it is non- invasive and requires neither insufflation nor sedation. Also, patients can continue normal daily activities immediately following the procedure. Thus, CCE is a possible alternative or complementary method to colonoscopy, and can be used concurrent with computed tomography colonography.

According to a recent systematic review and meta-analysis, second-generation colon capsule endoscopy (CCE2) detected polyps $>6 \mathrm{~mm}$ and $>10 \mathrm{~mm}$ with sensitivities of $86 \%$ and $87 \%$, respectively, and with specificities of $88 \%$ and $95 \%$, respective- 
ly [9]. The European Society of Gastrointestinal Endoscopy (ESGE) now recommends CCE as an alternative screening method for average-risk individuals [10]. In February 2014, CCE received US Food and Drug Administration clearance for use in patients following incomplete colonoscopy. Further studies are ongoing to evaluate CCE2 as first-line screening for colorectal cancer.

The diagnostic value of CCE is mostly dependent on the cleanliness of the colon. Indeed, CCE does not inherently have functions that allow washing of the mucosa during the procedure. Also, CCE often produces obstructed images because of bile, mucus, bubbles, food residue and blood clots. Adequate bowel preparation is mandatory to ensure a successful CCE, and the capsule must be excreted before the battery life has ended. Previously published studies show a CCE2 completion rate of roughly $93 \%$ [11]. While a high volume $(3-4 \mathrm{~L})$ of polyethylene glycol-electrolyte (PEG) is used as the primary laxative in most cases, the incorporation of prokinetics and laxative booster agents varies between centers. Adequate cleanness is achieved in only $61 \%$ to $81 \%$ of patients $[4,12,13]$. To date, there is no consensus concerning optimal bowel preparation for a CCE procedure [14-16]. One of the reasons for this controversy is that the currently used cleanliness grading systems have not been standardized. Therefore, a generally accepted grading system is not available for CCE. In the setting of small bowel video capsule endoscopy (SB-VCE), which has been used longer, multiple attempts to formulate a consensual grading system have been made but results so far have been disappointing [17-21]. These studies suggest grading scales should be focused on quantitative rather than qualitative criteria to achieve better reproducibility [21]. In 2011, Van Weyenberg et al. [17] used a computed quantitative scale to assess mucosal visibility during SB-VCE, based on a colorimetric index of the tissue color bar. We have developed a similar approach for SBVCE still frame analysis [22]. To our knowledge, this approach has not been described in the setting of CCE.

A consensual, user-friendly, reproducible grading scale would be helpful to assess quality of bowel preparation and for research purposes (in determination of the best preparation regimen by comparing regimens). The aim of this study was to evaluate two CAC scores allowing assessment of the quality of bowel preparation in CCE.

\section{Patients and methods}

This is a monocentric, prospective study with the objective of developing a CAC for CCE. The study was approved by the French Data Protection Authority (Comité National Informatique et Liberté) based on the principle that all images we deidentified.

\section{Inclusion criteria}

Eligible participants presented with an indication for CCE: incomplete colonoscopy, contraindication or refusal of conventional colonoscopy. CCE videos were edited so that only the part between the first image of the cecum and the last one of the rectum was kept for analysis. The videos were then converted into mpeg files and included in the study.

\section{Exclusion criteria}

Patients were excluded if the CCE was a first-generation colon capsule endoscopy (CCE1), if the procedure was incomplete, if the transit time was too rapid $(<5 \mathrm{~min})$, if lesions of any kind were observed, and if the video was judged to be too poor in quality after a first lecture by $A B$.

\section{CCE2 procedure}

Bowel preparation consisted of a clear liquid diet on the day prior to the procedure, followed by split ingestion of PEG lavage solution, $2 \mathrm{~L}$ the evening before and $2 \mathrm{~L}$ on the morning of the procedure day. After the capsule had exited the stomach, a booster consisting of $45 \mathrm{~mL}$ of sodium phosphate ( $\mathrm{NaP}$ ) was administered. Metoclopramide $(10 \mathrm{mg}$ ) could be administered orally if the capsule was delayed in the stomach ( $>1$ hour). The procedure was complete when the capsule was expelled (last image of the rectum identified). The second-generation capsule system used in this study consisted of the ingestible CCE2 and the Rapid $\mathrm{v} 8$ reading software (PillCam system, Medtronic).

\section{Capsule image computerized analysis and selection}

For every CCE2 video sequence, each still frame was individualized and de-identified. Color intensities in the red $(R)$, green (G) and blue (B) channels of each individual frame were identified using MATLAB R2014a software (MathWorks, Natick, USA) running on a commercially available solid-state hard drive (8 Go RAM, $2.9 \mathrm{GHz}$ Intel Core Processor). Our hypothesis, similar to that of Van Weyenberg et al. [15] at the tissue color bar level, was that a still frame showing good-quality preparation is associated with high values of red intensity and low values of green intensity (higher R/G ratio), whereas a still frame with poorquality preparation is associated with low values of red intensity and high values of green intensity (lower R/G ratio). We further surmised that since red and green added together produce a brown color, poor-quality still frames might also be associated with a high value of brown intensity (red+green). Each still frame was allotted a CAC score, based on the red and green color intensities, formalized as $R / G$ and $R /(R+G)$ ratios. Subsequently, all still frames were sorted by ratio value allowing distribution assessment. The still frames were divided in groups comprised in intervals (at least 16) of equal range, from the lowest to the highest CAC score value. Afterwards, for each CCE2 procedure, 12 still frames were randomly selected from each interval constituting a panel representative of the variety of CAC score values (i. e., image quality). This procedure was repeated twice, once for each ratio $(R / G$ and $R /(R+G))$. After sorting and random selection, two sets of still frames, deriving from 12 CCE procedures, representative of the range of these ratios, were obtained for each type of ratio. 


\section{Capsule image expert review}

The two sets of still frames were analyzed by two experienced capsule readers (XD and CF) who were blinded to the CAC values. The expert readers had previously analyzed over 500 capsule procedures. This review was performed twice for each set of still frames. Each set was shuffled between the two readings. Mucosal visualization of each still frame was determined based on an existing five-point grading scale described in the setting of SB-VCE by Brotz et al. [19], according to the percentage of visible mucosa; amount of fluid, bile, mucus, bubbles and food residue; and luminosity (Box). Still frames of excellent and good quality were classified as adequate, whereas still frames of fair and poor quality were classified as inadequate. Thus, each still frame was read four times (twice by each expert). In the event of discrepancies (twice classified as adequate and twice as inadequate), the experts studied the relevant still frames until an agreement was reached with regard to its classification.

\section{DEFINITION}

Definition of "adequate" and "inadequate" small bowel cleansing according to Brotz et al. [19].

Excellent: visualization of $\geq 90 \%$ of the mucosa, no or minimal fluid and debris, bubbles, and bile/chyme staining; no or minimal reduction of brightness

Good: visualization of $\geq 90 \%$ of the mucosa, mild fluid and debris, bubbles, and bile/chyme staining; mildly reduced brightness

Fair: visualization of $\leq 90 \%$ of the mucosa, moderate fluid and debris, bubbles, and bile/chyme staining; moderately reduced brightness

Poor: visualization of $\leq 80 \%$ of the mucosa, excessive fluid and debris, bubbles, and bile/chyme staining; severely reduced brightness

\section{Statistics}

Quantitative variables were reported in mean values. Qualitative variables were reported in percentage values. Pearson interobserver and intraobserver correlation coefficients were also calculated. ROC curves were fashioned for each type of ratio $[R / G$ and $R /(R+G)]$, with the experts reading as reference. Then, ratio cut-off values with the highest operating point (i.e., trade-off between highest sensitivity and highest specificity) were obtained, yielding the highest diagnostic performance in terms of discrimination between adequate and inadequate still frames. Sensitivity (primary endpoint) and specificity, positive predictive value (PPV) and negative predictive value (NPV) (secondary end points) of these cut-off values were then calculated.

\section{Results}

\section{Image selection and computed assessment of cleansing score feasibility}

Twelve CCE2 procedures were included after first analysis and editing. Of these video sequences, 79,497 still frames were extracted. The color intensity in the red and green channel was measured for all still frames. The green intensity ranged from 27.306 to 86.752 (mean 56.310) and the red intensity ranged from 34.613 to 124.280 (mean 79.200). All color measurements were repeated and yielded the exact same results (Pearson intra-test correlation coefficient of 1.0). Two computed scores were defined as the $R / G$ and the $R /(R+G)$ ratio, and then calculated for each still frame. The $R / G$ ratios ranged from 0.887 to 1.924 with a mean ratio of 1.411 . The $R /(R+G)$ ratios ranged from 0.470 to 0.658 with a mean ratio of 0.584 . Distribution of the ratio values was determined, and based on the range between the lowest and highest ratio value, equal intervals were created ( $\mathbf{F i g} \mathbf{1}$ ). Eighteen intervals of a 0.05 value were created for the $R / G$ ratios. Sixteen intervals of a 0.01 value were created for the $R /(R+G)$ ratios. Twelve still frames per interval per CCE2 procedure were then randomly selected and included in the datasets. All in all, 216 still frames were included in the $R / G$ set and 192 in the $R /(R+G)$ set.

\section{Still frame analysis}

The expert reading results are described in $>$ Table 1 . For the $\mathrm{R} / \mathrm{G}$ ratio, 160 still frames (74.1\%) were classified as inadequate and 34 (15.7\%) as adequate. Among the 22 (10.2\%) remaining still frames with discrepancy, 19 were classified as inadequate and 3 as adequate after analysis by both experts. For the $R /(R$ $+G)$ ratio, 161 still frames (83.8\%) were classified as inadequate and $16(8.3 \%)$ as adequate. Among the 15 (7.8\%) remaining still frames with discrepancy, 12 were classified as inadequate and 3 as adequate after analysis by both experts. Fig. 2 shows CCE2 still frames representative of different ratios. For the R/G set, Pearson intraobserver correlation coefficients were 0.66 for $X D$ and 0.75 for $C F$. For the $R /(R+G)$ set, Pearson intra-observer correlation coefficients were 0.88 for $X D$ and 0.75 for CF. The Pearson inter-observer correlation coefficient was 0.53 .

\section{Cut-off value and diagnostic performances}

A ROC curve was established for each ratio, with a different cutoff value per interval ( $>$ Fig. 3 ). The cut-off value of the R/G ratio with the best sensitivity and specificity in discriminating adequate from inadequate still frames was 1.55 . The sensitivity of this CAC score cut-off was $86.5 \%$ and the specificity $78.2 \%$. The PPV was $45.1 \%$ and the NPV was $96.6 \%$. The cut-off value of the $R /(R+G)$ ratio with the best sensitivity and specificity in discriminating adequate from inadequate still frames was 0.58 . The sensitivity of this CAC score cut-off was $95.5 \%$ and the specificity $63 \%$. The PPV was $25 \%$ and the NPV was $99 \%$. These results are summarized in $>$ Table 2 . 

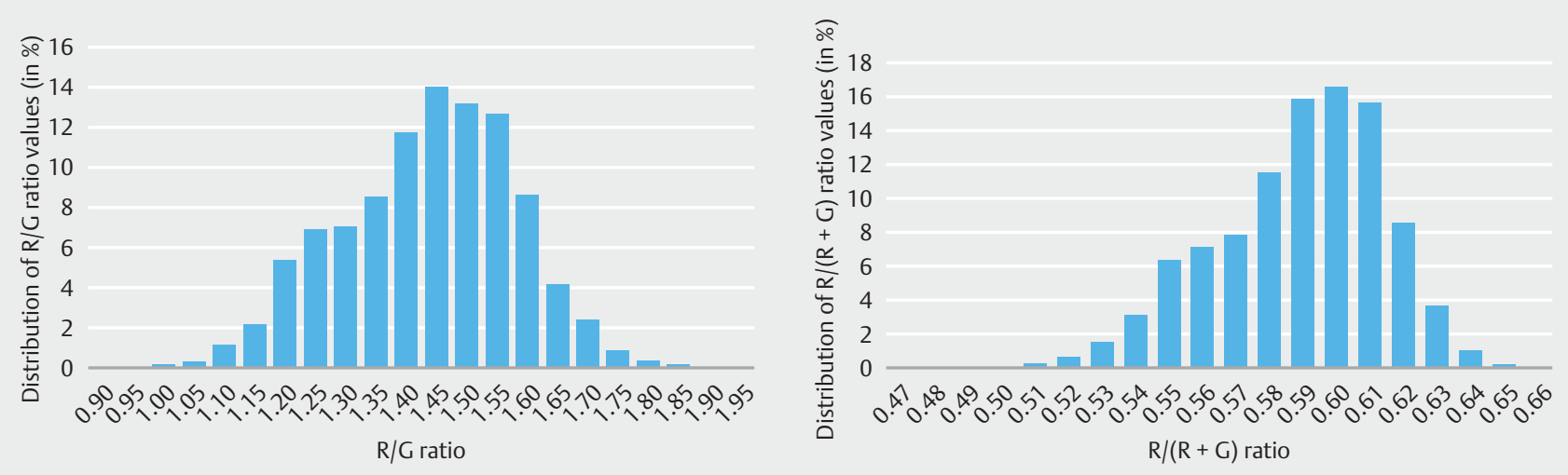

- Fig. 1 Colon capsule still frame computed assessment of cleansing (CAC) score distributions, for red over green (R/G ratio), and for red over brown $R /(R+G)$ ratio.

- Table 1 Results of the experts' readings and agreement of the two datasets of colon capsule still frames.

\begin{tabular}{|c|c|c|c|c|c|c|}
\hline Ratio & $\begin{array}{l}\text { Number of } \\
\text { frames }\end{array}$ & $\begin{array}{l}\text { Adequately } \\
\text { cleansed (\%) }\end{array}$ & $\begin{array}{l}\text { Inadequately } \\
\text { cleansed (\%) }\end{array}$ & Discrepancies (\%) & $\begin{array}{l}\text { Reclassified as } \\
\text { adequate }\end{array}$ & $\begin{array}{l}\text { Reclassified as } \\
\text { inadequate }\end{array}$ \\
\hline$R / G$ & 216 & $33(15.3 \%)$ & $161(74.5 \%)$ & $22(10.2 \%)$ & 3 & 19 \\
\hline$R /(R+G)$ & 192 & $16(8.3 \%)$ & $161(83.8 \%)$ & $15(7.8 \%)$ & 3 & 12 \\
\hline
\end{tabular}

- Table 2 Diagnostic performance of computed assessment of cleansing score, according to cut-off values of $R / G$ and $R /(R+G)$ ratios.

\begin{tabular}{|c|c|c|c|c|c|c|c|}
\hline Ratio & Cut-off value & Sensitivity (\%) & Specificity (\%) & PPV (\%) & NPV (\%) & $\begin{array}{l}\text { "Adequate" frames } \\
\text { among low ratio }\end{array}$ & $\begin{array}{l}\text { “Adequate" frames } \\
\text { among high ratio }\end{array}$ \\
\hline$R / G$ & 1.55 & 86.5 & 78.2 & 45.1 & 96.6 & $5 / 145$ & $39 / 71$ \\
\hline$R /(R+G)$ & 0.58 & 95.5 & 63.0 & 25.0 & 99.0 & $0 / 108$ & $65 / 84$ \\
\hline
\end{tabular}

\section{Calculation times}

Mean ( \pm standard deviation) $R / G$ and $R /(R+G)$ ratios calculation time were $1.893 \pm 0.042 \mathrm{msec}$ and $1.957 \pm 0.052 \mathrm{msec}$ per ratio per still frame, respectively.

\section{Discussion}

In this study, we developed two CAC scores based on the R/G or $R /(R+G)$ ratio calculation of individual still frames to evaluate mucosal visibility during CCE. Reproducibility of this scoring system was optimal, suggesting that an automated assessment of colon cleansing is feasible. We established a cut-off value for the $R / G$ ratio of 1.55 , with a sensitivity of $86.5 \%$ and a specificity of $77.7 \%$. CAC score based on the $R / G$ ratio performs better than $R /(R+G)$ in terms of its ability to discriminate adequate from inadequate still frames. R/G CAC score appears to be less effective in the colon than in the small bowel [17].
Cleanliness of the colon has a major influence on the diagnostic value of CCE [11]. Therefore, reporting quality of colon preparation is important to assessing reliability of CCE findings. It is also critical to have a reliable tool to assess quality of colon preparation to be able to compare different bowel-cleansing regimens in clinical trials [20]. However, the best method for assessment of bowel cleanliness in CCE has yet to be determined yet. Of note, in our study, despite use of a standardized scale, Pearson intraobserver correlation coefficients were good among our expert readers ( 0.66 to 0.88$)$, but the Pearson interobserver correlation coefficient was low (0.53). This argues in favor of lack of reliability and adequacy of semiquantitative assessment of still frames and that computerized evaluation might resolve this issue. In addition, in the setting of SB-VCE, development of a computed scoring system based on color intensities helped address these issues [17,22]. For instance, in 2011, Van Weyenberg et al. [15] published the first study using a computed quantitative scale to assess mucosal visibility dur- 

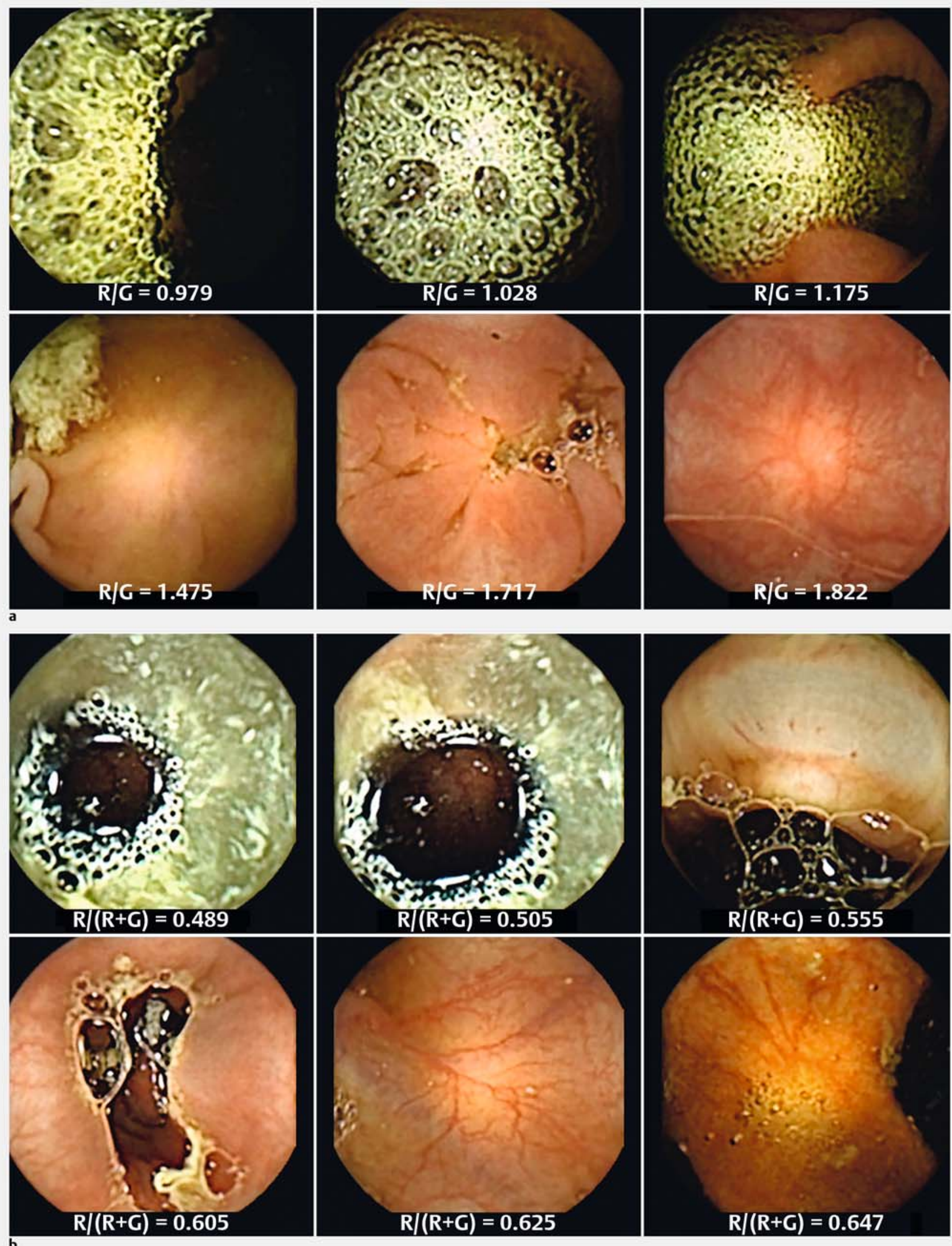

- Fig. 2 a Colon capsule still frames representative of different red over green (R/G) ratio. b Colon capsule still frames representative of different red over brown $(R /([R+G])$ ratio. 

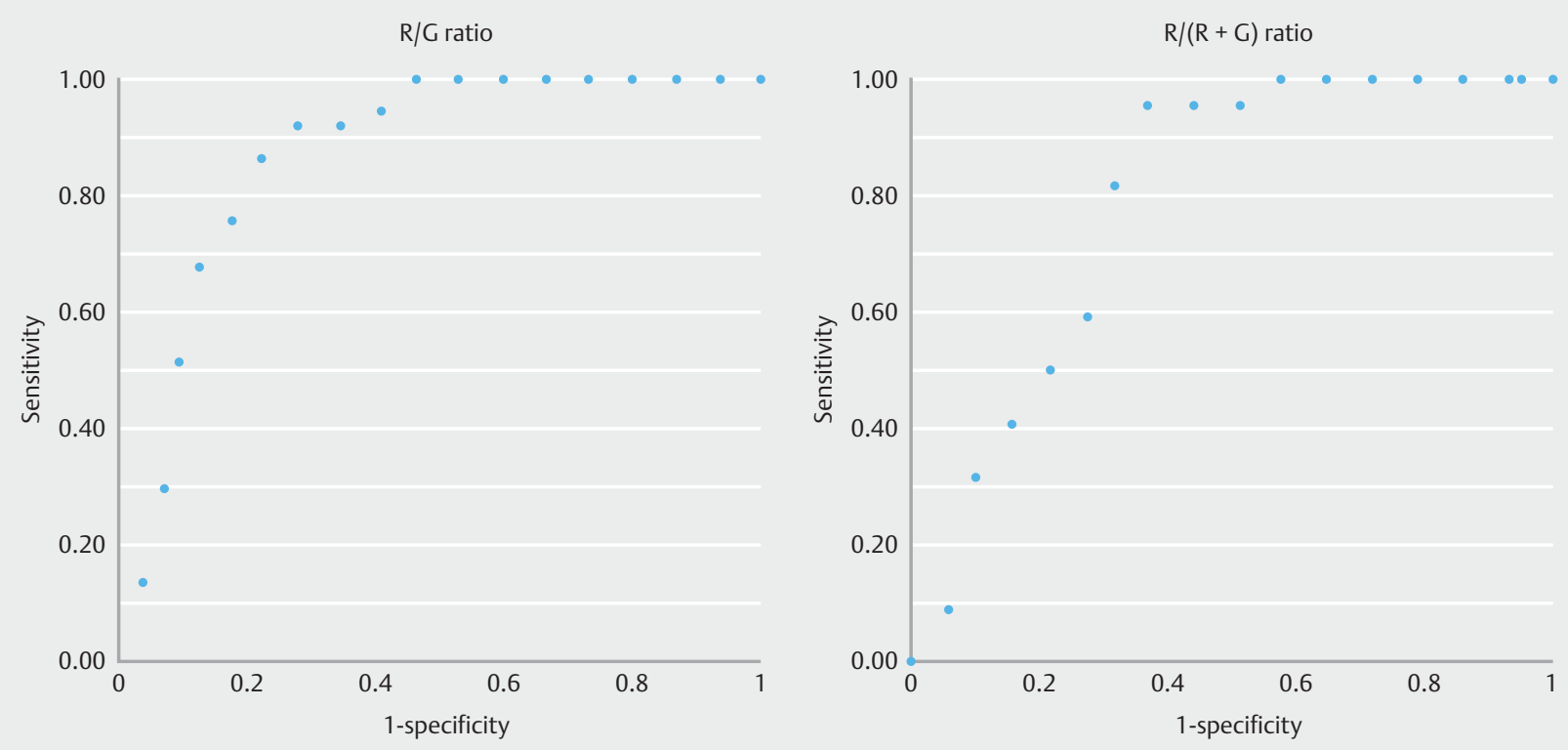

Fig. 3 Receiver operating characteristic curves of each Computed Assessment of Cleansing score distributions, for red over green (R/G) ratio, and for red over brown $R /(R+G)$ ratio.

ing SB-VCE. The results suggested that a computed scoring system based on color intensities in the red and green channel of the tissue color bar could be used to assess quality of cleansing. There was strong agreement between the computed scale (CAC) and previously reported scales for assessment of quality of small-bowel preparation. Our group has shown that the CAC score was feasible, objective, reproducible, and even more sensitive $(94 \%)$ and specific $(88 \%)$ when based on color intensities in the red and green channel at the image level [22]. We decided to use this approach, at the image level, in the setting of CCE.

Some strengths of this study should be emphasized. We constructed a robust method of analysis of the quality of bowel preparation, which was then used to evaluate both CAC scores. To start with, only second-generation CCE procedures were included in the study. Then, analysis of a significant number (408) of still frames was performed, twice, in a random fashion, by two experienced capsule readers blinded to the ratio values, using a standardized and precise scale. This allowed reliable clinical assessment of quality of cleansing in the still frames. As a result, our findings were consistent with that of other similar studies, in terms of reproducibility and Pearson interobserver and intraobserver correlation coefficients $[15,22]$, which points to good external validity. Worth mentioning, the time of extraction of color intensities and calculation of the ratios were very low (less than 2 msec per still frame using standard software on a commercially available computer system). This signifies that the computed score has a good applicability, as it is easy and rapid to calculate, with simple tools. We can expect it to be applicable to full-length videos and also to be low cost to develop.

Several limitations of the study also must be discussed. First, we included only complete and normal CCE video recordings.
That decision was based on the fact that incomplete and abnormal CCE exams would, in clinical practice, always warrant further diagnostic and/or therapeutic procedures (i. e., colonoscopy in most cases). Subsequently, these CCE exams are not the ones for which a cleaning score is needed most. The second limitation, similar to what our group has done for development of a CAC score for SB-VCE [22], was that we worked on still frames rather than full-length videos. Colorimetric analysis of the tissue color bar, proposed for use on videos by Van Weyenberg et al., [15] is extremely convenient and certainly gives clues but it entails a major loss of data as compared to what is readily available by using images. Moreover, color analysis, albeit an important image characteristic, represents only a limited portion of what can be analyzed from an image. Other characteristics, such as presence of bubbles, luminosity, and contrast, can be extracted from still frames and could contribute to improving the diagnostic performance of the score. These characteristics would not be extractable from the tissue color bar. Therefore, we used a significant number (408) of still frames, whereas we considered the limited number (12) of videos used for this study of little importance. The main drawback of this approach is that we cannot draw definitive conclusions on the performance of the R/G CAC algorithm when applied to full-length videos. In the future, full-length CCE videos should be analyzed on a frame-by-frame basis, and a cut-off score value for an adequate video will have to be determined. Third, expert review of still frames was based on a grading scale described in the setting of SB-VCE. This scale was used because its robustness has been proven for analysis of SB still frames, a validated scale for colon still frame analysis is lacking, and this qualitative scale assesses elements (percentage of visible mucosa, amount of fluid, bile, mucus, bubbles and food residue, 
and luminosity) that we believe are correlated to still frame adequacy in the colon.

\section{Conclusion}

Determination of mucosal visibility, much like what is done with the Boston Bowel Preparation Scale in colonoscopy, is of capital importance in the setting of CCE procedures. We showed that a CAC score to assess the quality of bowel preparation based on a color intensity ratio of red and green pixels on still images is feasible, rapid, and reproducible. Such a score may circumvent the subjectivity of qualitative grading systems. To our knowledge, it is the first score of this type to have been tested in $C C E$. The $R / G$ ratio used, which has shown promising results in SB-VCE [22], does not allow sufficient discrimination between adequate and inadequate still frames (good sensitivity, mediocre specificity) in CCE and therefore must be modified. More parameters should be included in the algorithm, such as other color intensity ratios, contrast, homogeneity, and uniformity. Its performance must be enhanced and assessed at the video level so that, in the future, it can be used as a substitute for subjective scales and to compare different bowel preparation regimens in clinical trials.

\section{Acknowledgements}

A computer equipped with the software Rapid was lent by Medtronic.

Competing interests

Xavier Dray has acted as a consultant for Boston Scientific, Fujifilm, Pentax and Medtronic. Marine Camus has acted as a consultant for Boston Scientific.

\section{References}

[1] Koulaouzidis A, Rondonotti E, Karargyris A. Small-bowel capsule endoscopy: a ten-point contemporary review. World J Gastroenterol 2013; 19: $3726-3746$

[2] Fisher LR, Hasler WL. New vision in video capsule endoscopy: current status and future directions. Nat Rev Gastroenterol Hepatol 2012; 9: $392-405$

[3] Rondonotti E, Soncini M, Girelli C et al. Small bowel capsule endoscopy in clinical practice: a multicenter 7-year survey. Eur J Gastroenterol Hepatol 2010; 22: $1380-1386$

[4] Eliakim R, Yassin K, Niv Y et al. Prospective multicenter performance evaluation of the second-generation colon capsule compared with colonoscopy. Endoscopy 2009; 41: 1026-1031
[5] Adrián-de-Ganzo Z, Alarcón-Fernández O, Ramos L et al. Uptake of colon capsule endoscopy vs colonoscopy for screening relatives of patients with colorectal cancer. Clin Gastroenterol Hepatol Off Clin Pract J Am Gastroenterol Assoc 2015; 13: 2293 -2301.e1

[6] Spada C, Hassan C, Barbaro B et al. Colon capsule versus CT colonography in patients with incomplete colonoscopy: a prospective, comparative trial. Gut 2015; 64: 272 - 281

[7] Spada C, Hassan C, Costamagna G. Colon capsule endoscopy. Gastrointest Endosc Clin N Am 2015; 25: 387-401

[8] Pioche M, de Leusse A, Filoche B et al. Prospective multicenter evaluation of colon capsule examination indicated by colonoscopy failure or anesthesia contraindication. Endoscopy 2012; 44: 911 - 916

[9] Spada C, Pasha SF, Gross SA et al. Accuracy of first- and second-generation Colon capsules in endoscopic detection of colorectal polyps: a systematic review and meta-analysis. Clin Gastroenterol Hepatol Off Clin Pract J Am Gastroenterol Assoc 2016; 14: 1533 - 1543

[10] Spada C, Hassan C, Galmiche JP et al. Colon capsule endoscopy: European Society of Gastrointestinal Endoscopy (ESGE) Guideline. Endoscopy 2012; 44: 527-536

[11] Van Gossum A, Munoz-Navas M, Navas MM et al. Capsule endoscopy versus colonoscopy for the detection of polyps and cancer. N Engl J Med 2009; 361: $264-270$

[12] Spada C, Hassan C, Munoz-Navas M et al. Second-generation colon capsule endoscopy compared with colonoscopy. Gastrointest Endosc 2011; 74: $581-589 . e 1$

[13] Morgan DR, Malik PR, Romeo DP et al. Initial US evaluation of secondgeneration capsule colonoscopy for detecting colon polyps. BM] Open Gastroenterol 2016; 3: e000089

[14] Ramos L, Alarcón O, Adrian Z et al. One-day versus two-day cleansing for colon capsule endoscopy: a prospective randomized pilot study. Gastroenterol Hepatol 2014; 37: $101-106$

[15] Van Weyenberg SJB, De Leest HTJI, Mulder CJJ. Description of a novel grading system to assess the quality of bowel preparation in video capsule endoscopy. Endoscopy 2011; 43: 406-411

[16] Fireman Z. What is the optimal bowel preparation for capsule endoscopy? Nat Clin Pract Gastroenterol Hepatol 2008; 5: 418-419

[17] Spada C, Riccioni ME, Familiari P et al. Polyethylene glycol plus simethicone in small-bowel preparation for capsule endoscopy. Dig Liver Dis Off J Ital Soc Gastroenterol Ital Assoc Study Liver 2010; 42: 365 370

[18] Park SC, Keum B, Hyun JJ et al. A novel cleansing score system for capsule endoscopy. World J Gastroenterol 2010; 16: 875-880

[19] Brotz C, Nandi N, Conn M et al. A validation study of 3 grading systems to evaluate small-bowel cleansing for wireless capsule endoscopy: a quantitative index, a qualitative evaluation, and an overall adequacy assessment. Gastrointest Endosc 2009; 69: 262 - 270, 270.e1

[20] Rex DK, Petrini JL, Baron TH et al. Quality indicators for colonoscopy. Gastrointest Endosc 2006; 63: S16-S28

[21] Bouchard S, Ibrahim M, Van Gossum A. Video capsule endoscopy: perspectives of a revolutionary technique. World J Gastroenterol 2014; 20: 17330 - 17344

[22] Abou Ali E, Histace A, Camus M et al. Development and validation of a highly sensitive and highly specific computed assessment of cleansing score for small bowel capsule. Endosc Int Open 2018; 06: E646 - E651 